\title{
Intracellular trafficking of diphtheria toxin and its mutated form, CRM197, in the endocytic pathway
}

\author{
Bilge Ozerman Edis, ${ }^{1}$ Ebru Haciosmanoglu, ${ }^{2}$ Basak Varol, ${ }^{1}$ Muhammet Bektas ${ }^{1}$ \\ ${ }^{1}$ Department of Biophysics, Istanbul University, Istanbul Faculty of Medicine, Istanbul, Turkey \\ 2Department of Physiology, Istanbul Bilim University, Faculty of Medicine, Istanbul, Turkey
}

\begin{abstract}
OBJECTIVE: Diphtheria toxin (DTx) is a well-characterized bacterial toxin. However, the endocytic pathway of the mutant of DTx, CRM197, which is used as an immunological adjuvant, has not yet been fully explained. The aim of this study was to investigate the intracellular trafficking of CRM197-loaded endosomes.

METHODS: Human umbilical vein endothelial cells (HUVECs) were used in a cell culture. The effective incubation time was determined by transmission electron microscopy in toxin-treated cells. Density gradient centrifugation and ADP-ribosylation assay were used to isolate and detect toxin-loaded endosomal fractions. Endosomal fractions from CRM197-treated cells were elicited after 15 minutes of incubation and the presence of fragment A was demonstrated using Western blot. Immunofluorescence microscopy was used to identify endosomes in CRM197-treated endothelial cells.

RESULTS: DTX-loaded endosomes were detected as enlarged vesicles in the perinuclear area with 15 minutes of toxin treatment. DTx-loaded endosomal fractions were determined by ADP-ribosyltransferase activity test and Western blot analysis. Enzymatic activity of the toxin-loaded endosomal fraction increased by $20 \%$ in actin cytoskeletal-damaged cells treated with cytochalasin D. The steps for the toxin treatment of HUVECs with DTx and obtaining endosomal fractions were repeated for CRM197. In the CRM197-loaded endosomal fraction, actin and Hsp90 were identified in addition to fragment A. Fluorescent images revealed that CRM197-loaded endosomes were co-localized with actin filaments and that Rab11, which signals the return to the plasma membrane, was more prominent than Rab7, the lysosomal pathway indicator.
\end{abstract}

CONCLUSION: These results suggest that CRM197-loaded endosomes participate in the recycling pathway.

Keywords: Actin cytoskeleton; CRM197; diphtheria toxin; endosome; Human umbilical vein endothelial cells.

Cite this article as: Ozerman Edis B., Haciosmanoglu E., Varol B., Bektas M. Intracellular trafficking of diphtheria toxin and its mutated form, CRM197, in the endocytic pathway. North Clin Istanb 2018;5(2):89-95.

$\mathrm{D}$ iphtheria toxin (DTx) is a well- characterized bacterial toxin, which induces inhibition of protein synthesis by ADP-ribosylation of eukaryotic elongation factor 2 (eEF2). In the presence of specific receptors, DTx enters into cells via endocytosis, and passes into cytosol then ADP-ribosylates eEF2 over histidin (715. amino acid). Inhibition of protein synthesis, destruction of actin cytoskeleton and activation of nuclease are fol- lowed by induction of apoptotic process in the cell [1]. However mutant forms of diphtheria toxin (CRM197, CRM228) cannot inhibit protein synthesis [2].

Among cross-reactive mutants, CRM197 is used as a carrier molecule in pediatric conjugated vaccines [3]. Mutant diphtheria toxin CRM197 which functions as carrier protein in vaccines developed against bacterial infections, and also used for the construction of immuno- 
logical adjuvant has the same number $(n=535)$ of amino acids with natural toxin. Because of a single amino acid substitution in fragment A (FA) CRM197 carries glutamic acid in position 52 instead of glycine [4]. DTx enters into cell, ADP-ribosylates eEF2 with its FA component in the presence of NAD, and triggers inhibition of protein synthesis, and activation of apoptotic pathways [5]. Mutation in CRM197 induces loss of enzymatic activity of FA so CRM197 does not lead to any change in intracellular protein synthesis. CRM197 enters into cell via binding of its fragment $\mathrm{B}(\mathrm{FB})$ to $\mathrm{DT}$ x receptor. The precursor (pro-HB-EGF) of heparin-binding epidermal growth factor like growth factor (HB-EGF) anchored on the cell surface functions as a receptor for the toxin [6]. Varol et al. have presented endocytosis of DTx, and stages of activation of FA in cytosol in detail [1]. Currently, diverse hypotheses have been suggested concerning exit of FA from endosome, and its way of access into targeted region. According to a supportive model, entry of FA into cytosol requires presence of compounds as, Hsp90 chaperone, cytosolic translocation factors as thioredoxin reductase 1 , and cytosolic factors as ATP and $\beta$-COP [7]. In addition to cytosolic translocation factors, eEF2 which is one of the components of protein synthesis mechanisms, and actin from components of cytoskeleton have been found to be effective in delivery of FA of both DTx and mutant CRM197 through endosomes into cytosol [8]. In studies performed on DTx, and CRM197, it has been determined that as a result of interactions between FA-eEF2-actin, ADP-ribosylation of eEF2 occurs, but actin filaments are also depolymerized, and finally destroyed [9]. Just as the case in FAactin interaction, under the impact of CRM197, actin filaments are also depolymerized [10]. From molecular dynamics simulations approach (homology modelling) the potential interaction site between FA of DTx and actin has been determined [11]. Loss of intercellular contacts was observed as a result of depolymerization of actin filaments of endothelial cells incubated with CRM197 for 24 hours in cell culture media [12]. In the same study the interaction between CRM197, and $\mathrm{G}$-actin was determined using chromatographic methods, and as a result of thermodynamic calculations of molecular dynamics simulations an interaction interface was proposed. On the interaction interface, three areas where amino acids in CRM197 (lysin 48, cysteine 218 , and cysteine 233) may interact with respective amino acids of G-actin (glycin 197, arginin 62, and serine 60) were determined. Besides limited number of studies have been performed concerning intracellular pathway of CRM197.

As is also known, actin cytoskeleton supports intracellular movements of endosomes. Besides, interactions between actin and toxin tend to direct intracellular trafficking of CRM197. Endosomal migration signal, a process regulated by Rac-GTP, is transferred to actin cytoskeleton by Rab5 signal peptide carrying early endosomes [13]. Late endosomal Rab7 transmits the signal of cargo digestion, while Rab peptides in recycling (Rab4, Rab11) direct endosomes to plasma membrane. Endosomes carrying Rab4 signal peptide on their surface rapidly, but endosomes loaded with signal peptide Rab11 slowly fuses with plasma membranes [13]. For the delivery of toxin into cytosol endosomes attach Tdomain which is identified with 200-387 amino acids of FB part of the toxin transported into the cell, then the toxin is subjected to proteolytic degradation. [14]. Following this stage, the pathway of endocytic process for CRM197 has not been definitively identified yet. In this study, in order to investigate the pathway selected by CRM197-loaded endosomes toxin infected cells were examined. Firstly DTx was used to the determine application time of CRM197 then ultrastructural analysis and ADP-ribosylation assay were carried out in toxin loaded endosomes. Incubation of cell suspension was limited to 15 minutes so as to obtain CRM197 loaded endosomes. Western Blot analysis determined that within this specified time interval FA of CRM197 was not delivered from endosomes into cytosol. Immunofluorescent labeling detected that Rab 11 peptide in early endosomes of CRM197 treated cells exposed a more powerful fluorescent signal than other Rab peptides.

\section{MATERIALS AND METHODS}

\section{Chemicals}

In this study anti-Rab7 (Sigma), anti-Rab11, and anti-Hsp90 (Abcam), anti-Actin (Santa Cruz), anti-FA (7F2) (Abcam), and anti-EEA1 (Abcam) were used as primary antibodies. Secondary antibody, and fluorescent (TRITC) labeled phalloidin were purchased from Santa Cruz, and Sigma, culture materials from Nunc, and Falcon firms, DTx, and CRM197 from Calbiochem, and ProLong Gold Antifade Mountant Invitrogen firms. For ADP-ribosylation assay [Adenosine $-{ }^{14} \mathrm{C}$ ] NAD (NENDupont) with a specific activity of $535 \mathrm{Ci} / \mathrm{mol}$ was used. 


\section{Cell culture}

Human umbilical vein endothelial cells (HUVECs) were used in the study. Under standard culture conditions, Dulbecco's Modified Eagle's medium (DMEM F-12) which contained 10\% Fetal Bovine Serum (FBS) and antibiotic $(100 \mu \mathrm{g} / \mathrm{ml}$ streptomycin, and $100 \mathrm{U} /$ $\mathrm{ml}$ penicillin) was used, and cellular proliferation was achieved at $37^{\circ} \mathrm{C}$ with a $5 \% \mathrm{CO}_{2}$ content. Trypan blue was used for viability of cells which were incubated on 6 -well plates $\left(1 \times 10^{6}\right.$ cell $\left./ \mathrm{ml}\right)$ containing coverslip, and treated with CRM197 (0.8 nM) for 15 minutes.

\section{Ultrastructural analysis}

DTx $(0.8 \mathrm{nM})$-treated cells, and control cells were incubated at $37^{\circ} \mathrm{C}$, and on $5 \% \mathrm{CO}_{2}$ containing culture media for 15 minutes to make fine structural examination using electron microscope (TEM). Cells $\left(3 \times 10^{6}\right.$ cells/ $\mathrm{ml}$ ) were washed, with phosphate-buffer saline (PBS) and centrifugated (2000 rpm, for 3 minutes). Cells in pellet were fixed in $2.5 \%$ glutaraldehyde, and $1 \%$ osmium tetroxide. Cells were wrapped with egg white, and left overnight in $70 \%$ alcohol to harden them. After routine dehydration processing in a graded alcohol series, cells were blocked, and embedded in Epon ${ }^{\oplus}$ resin. Small slices were cut from blocks using ultramicrotome (C. Reichert OM U3), and transferred on copper grids. Grids were stained with contrasting agents as uranyl acetate and Reynolds solutions, and were examined with TEM (JEOL JEM 1011).

\section{Isolation of endosomes}

HUVECs cell suspension was incubated in the presence of CRM197 (0.8 nM) for 15 minutes. Endothelial cells $\left(10^{7}\right.$ cells $\left./ \mathrm{ml}\right)$ were precipitated while centrifuging them at $4^{\circ} \mathrm{C}$, and $270 \times \mathrm{g}$ for 5 minutes. The cells were suspended in PBS, and precipitated again. Cells in the pellet were suspended in $1 \mathrm{ml}$ homogenization buffer $(0.25 \mathrm{M}$ sucrose, $3 \mathrm{mM}$ imidazole, $0.5 \mathrm{mM}$ potasium4,6-diamidino-2-phenylindole (K-EDTA) $\mathrm{pH}: 7.3)$, and centrifuged at $750 \times \mathrm{g}$ for 7 minutes. The precipitated cell fraction was lysed in the same homogenization buffer $(200 \mu \mathrm{L})$ using insulin syringe [15]. The lysate was centrifuged at $750 \times \mathrm{g}$ for 7 minutes. The lysate pellet obtained was suspended in homogenization buffer $(500 \mu \mathrm{l})$ (17\% Percoll, and 10\% Iodixanol-Opti-Prep kit). A 17\% Percoll phase on the $65 \%$ sucrose bed added into ultracentrifuge tube, suspension $(500 \mu \mathrm{l})$ was layered. At the end of 1 hour-long precipitation process at $59,000 \times \mathrm{g}$ using
Sorvall AH 650 rotor, endosomal fraction was pipetted over Percoll phase.

\section{ADP-ribosylation assay}

DTx $(0.8 \mathrm{nM})$ treated cells were used to determine ADPribosyltransferase activity of FA-loaded endosomal fractions in the absence or in the presence of cytochalasin D, a membrane permeable compound causing the disruption of actin filaments [8]. ADP-ribosylation assay was realized at $20^{\circ} \mathrm{C}$ within 10 minutes in the presence of 25 $\mu \mathrm{l}$ reaction mixture containing $50 \mathrm{mM}$ Tris- $\mathrm{HCl}, \mathrm{pH}$ : 7, 4, $7 \mathrm{mM}$ 2-mercaptoethanol, $5 \mu \mathrm{M}$ [Adenosin $-{ }^{14} \mathrm{C}$ ] $\mathrm{NAD}$, in addition to $20 \mathrm{pmol} \mathrm{eEF} 2$. After incubation, 5 $\mu l$ samples were passed through GF/A Whatman filters, and washed with cold TCA. After drying, the filters were transferred to vials containing $5 \mathrm{ml} \mathrm{2,5-difenyloxalose}$ (PPO) in toluene. Radioactivity was determined in a liquid scintillation counter (Packard Tri-Carb1000 TR). Endosomal fractions were isolated in the presence of $1 \%$ Triton X-100 (400 $\mu \mathrm{l})$. For ADP-ribosylation assay 20 $\mu \mathrm{l}$ of sample, and for electrophoresis $10 \mu \mathrm{l}$ of sample (20 $\mu \mathrm{g}$ endosomal protein mixture) were used.

\section{Electrophoretic analysis}

Sodyum dodecyl sulphate-polyacrilamide gel electrophoresis (SDS-PAGE) was performed based on Laemmli method [16]. For determination of molecular weight standards prestained $10-170 \mathrm{kDa}$ protein kit (Page Ruler, Pierce) was used. Endosomal proteins subjected to electrophoretic analysis were stained with Coomassie brilliant blue, and excess stain was removed by solution containing $10 \%$ acetic acid, and $5 \%$ methanol.

\section{Western Blot}

After electrophoresis, proteins loaded gel was used for Western blotting. For this procedure SDS-PAGE gel was placed on nitrocellulose membrane, and between 3MM Whatman filter papers. This sandwich system was saturated with transfer buffer in semi-dry transfer device. Proteins were transferred onto nitrocellulose membrane at 150-200 mA, and lasted for half an hour. The membrane was incubated with Tris Buffered Salt Solution containing $5 \%$ bovine serum albumin to prevent nonspecific bindings so as to saturate the membrane. Membrane was washed twice with TBST solution, and incubated under room temperature for two hours with primary monoclonal antibody solution. Membrane was washed 
three times with TBST solution, then it was incubated for one hour under room temperature in the presence of alkaline phosphatase conjugated secondary Ig- $\mathrm{G}$ antibody. Membrane was rewashed three times with TBST solution, under room temperature, it was incubated at dark for the formation of bands in the presence of alkaline phosphatase substrate BCIP $(150 \mu \mathrm{g} / \mathrm{ml})$, and NBT $(300 \mu \mathrm{g} / \mathrm{ml})$. Membrane was placed in $20 \mathrm{mM}$ EDTA, and the reaction was stopped. Bands in nitrocellulose membrane were analyzed.

\section{Immunofluorescence microscopy}

During fluorescence microscopic examination, actin cytoskeleton was labeled with phalloidin-TRITC in mutant toxin-treated cells. CRM197 was identified with anti-FA. Anti-Rab4 was used for early endosome marker that regulates classification of endosomes in cells, and return of the receptor to the cell membrane, for late endosomes anti-Rab7, and for endosomes in the return cycle anti-Rab11 was used. Visualization of antibodies was ensured using anti-mouse or anti-rabbit IgG-FITC. Intracellular location of CRM197-treated cells was visualized using primary anti-FA antibody, and secondary anti-mouse IgG-FITC antibody. Cell nuclei were labeled using 4,6-diamidino-2-phenylindole (DAPI). For immunofluorescence microscopy cells on coverslip were washed with PBS, and incubated in $0.01 \%$ Triton $\mathrm{X}-100$ for 10 minutes. For fixation, the cells were incubated in $2 \%$ paraformaldehyde/PBS media at $4 \mathrm{oC}$ for 1 hour. Then primary antibodies diluted with $1 \%$ PBSBSA were incubated for 2 hours. Fluorescence labeled secondary antibodies were diluted in $1 \%$ PBS-BSA at a ratio of 1:2000, and incubated for one hour. Slides were examined under oil immersion objective of fluorescence microscope with triple filter (Olympus BX51) at 100x magnification. The images were photographed using Olympus DP-72 camera system, and DP2-TWAIN software program.

\section{RESULTS}

DTx untreated (control) and treated endothelial cells were examined under TEM for their ultrastructural details, and sizes of endosomes were compared (Fig. 1A). Increase in the diameter of early endosomes was detected (Fig. 1B). Increase in the size of endosomes of the endothelial cells at the end of 15 minutes of incubation was consistent with previous findings in literature. Following this time of DTx incubation, endosomal fractions were obtained with density gradient centrifugation (Figure 2 ). Endosomal fractions were prepared from cells in the absence or presence of cytochalasin D then ADP-ribosyltransferase activity was analyzed. In cases where actin
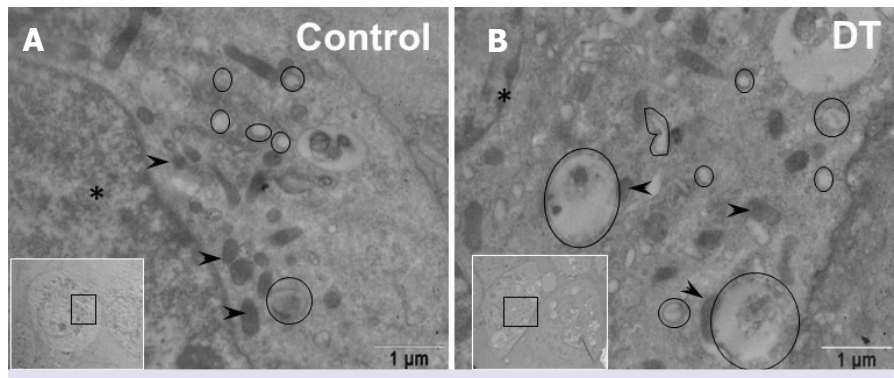

FIGURE 1. Transmission electron micrographs of endothelial cells: The magnified part of the cells was indicated in the lower left checkbox. When compared with the endosomes of the cells of control group (A) number, and size of the endosomes in diphtheria toxin-loaded cells markedly increased (B) (magnification 20,000x). On the micrograph some endosomes are shown in rings, while nuclei and mitochondria with asterixes $(*)$, and $(\bullet)$ signs, respectively (Scale bar: $1 \mu \mathrm{m}$ ).

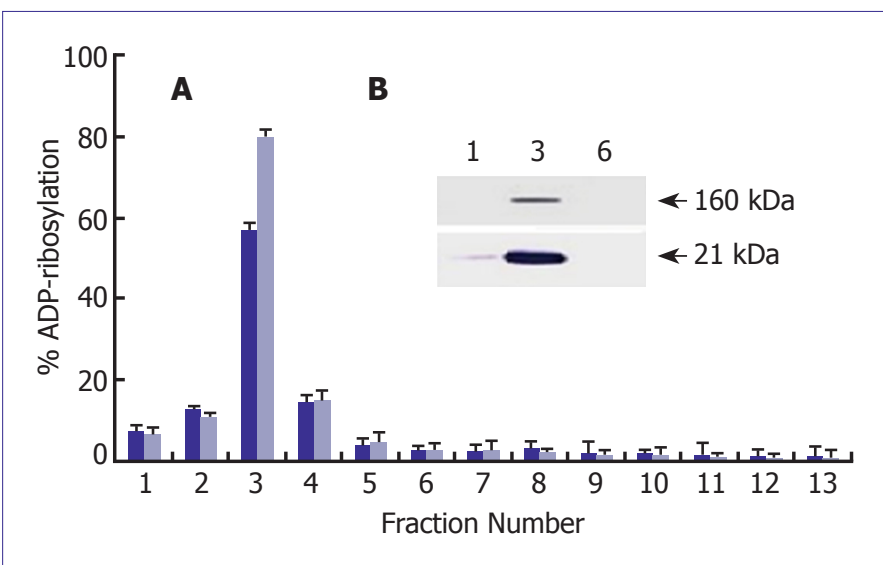

FIGURE2. Examination of endosomal fractions obtained from DTx -treated cells. (A) The level of ADP-ribosyltransferase activity of endosomal FA was shown in diphtheria toxintreated cells (dark-colored columns) or in cytochalasin $\mathrm{D}$ $(2 \mu \mathrm{M})$ added cells before toxin treatment (light-colored columns). Assay of ADP-ribosylation was repeated three times. (B) In the Western Blot analysis, anti- EEA1 (160 $\mathrm{kDa})(1: 500)$, and anti-FA (21 kDa) (1:500) were used to determine early endosomes and FA respectively in DTXtreated cells incubated previously with cytochalasin D. FA was detected in endosomes obtained from the 3rd fraction which had the highest ADP-ribosyl transferase activity. 


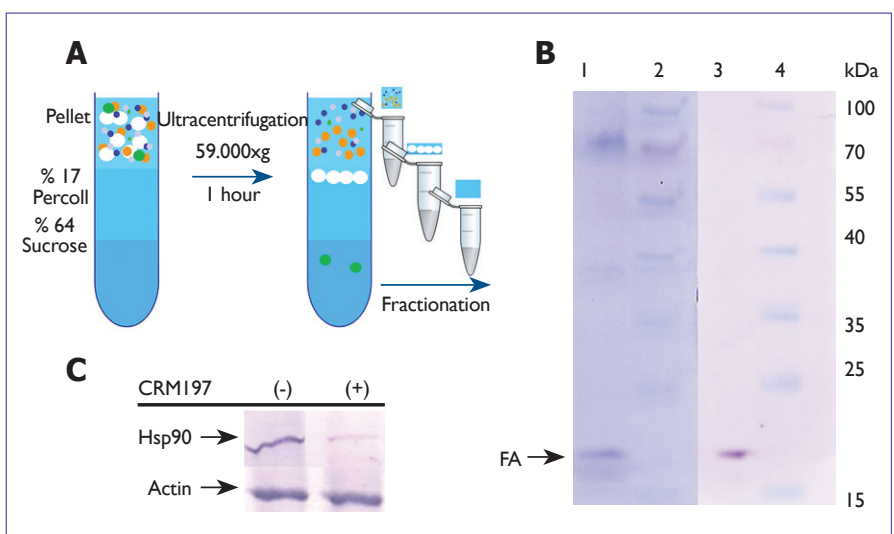

FIGURE 3. Western Blot analysis of endosomal fractions obtained from CRM197 treated cells. Endosomal fractions were obtained using density-gradient centrifugation (A). Endosomal fractions were observed with SDS-PAGE, and transferred on nitrocellulose membrane (B). Endosomal proteins on gel (line \#1) were identified using Coomassie brilliant blue dye and FA on membrane was labelled with anti-FA (1:200) (line \#3). Colored protein standard solutions are seen in lines \# 2, and 4. Anti-actin antibody (1:100), and anti-Hsp90 beta antibody [5G4] (1:1000) were used to identify actin and Hsp90 in endosomal fractions of CRM197 treated $(+)$ and untreated $(-)$ cells $(C)$.

cytoskeleton was damaged, enzymatic activity of the FAloaded endosomal fractions was detected $20 \%$ higher (Fig. 2A).

FA-loaded early endosomes were detected at the 3rd. fraction significantly in Western Blot analysis (Fig. 2B). Thus, it was determined that the FA had not yet completely delivered from endosomes into the cytosol of
DTx-treated cells and the endosomal fraction was still cargo-loaded within this incubation time period. Starting from these findings we concluded that for isolation of endosomal fraction from CRM197-treated cells, and follow-up of endocytic process, it should be appropriate to limit the incubation time to 15 minutes.

As indicated in the 'Method' section, early endosomes were obtained from CRM197-treated endothelial cell lysate (Fig. 3). At the last stage of isolation of early endosomes, density gradient centrifugation was used (Fig. 3A). CRM197-loaded early endosomes were determined by FA labeling in Western Blot analysis of endosomal fractions (Fig. 3B). On the other hand, Western Blot method was also used to determine the presence of actin, and Hsp90 that support the entry of cargo molecule carried with endosomes into cytosol (Fig. 3C).

Cells were incubated with CRM197 in culture media for 15 minutes so as to analyze intracellular trafficking of mutant toxin. Intracellular distribution of CRM197 was showed using immunofluorescence method (Fig. 4). FA of CRM197 was labeled and widespread distribution of CRM197 in endothelial cells was determined (Fig. 4A). Actin filaments of cells were labeled with phalloidin-TRITC so as to make actin cytoskeleton visible (Fig. 4B). As a result of triple labeling overlapping FA and actin filaments were observed (Fig. 4C). Endosomal markers and actin filaments were labeled to determine the intracellular pathway followed by CRM197-loaded endosomes. In control (Fig. 5A) and CRM197-treated cells (Fig. 5B) endosomal marker Rab11of the recycling pathway which regulates return of receptors onto plasma
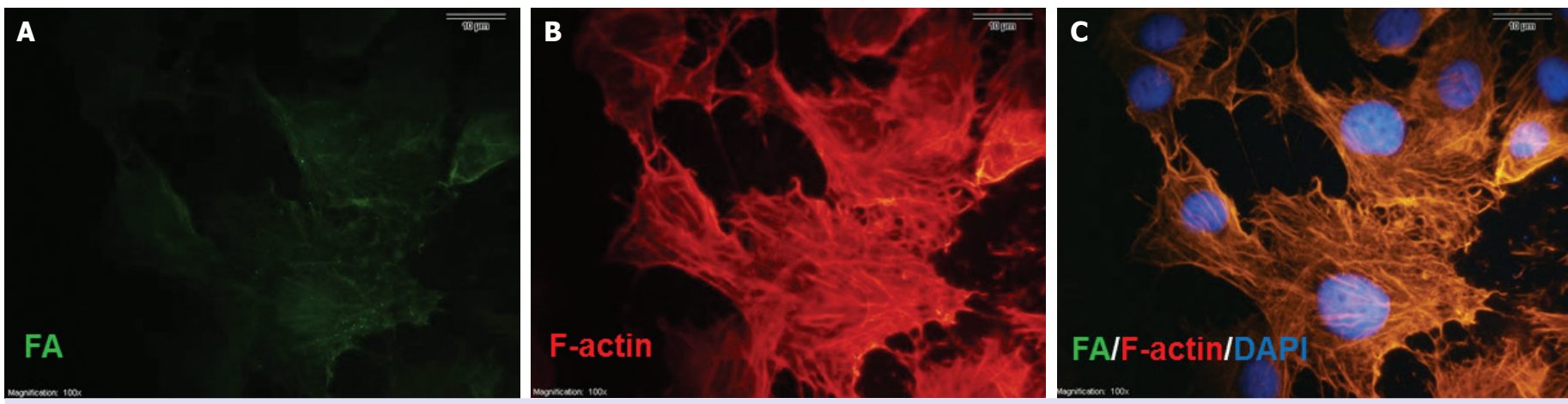

FIGURE 4. Intracellular trafficking of CRM197. Endothelial cells were treated with CRM197 (0.8 nM) for 15 minutes. The cells fixed on the slides to identify FA (green) were incubated with anti-FA monoclonal 7F2 primary antibody (1:1000) for 2 hours, and marked with FITC-labeled secondary antibody specific to this antibody (1:2000) (A). TRITC-labeled phalloidin (1:1000) was added into wells, and incubated for 1 hour to label actin cytoskeleton (red) (B). Cell nuclei (blue) were labeled with DAPI. Prepared slides were covered with ProLong Gold Antifade (C). As is seen in Figure 4, CRM197 is widely distributed in HUVEC, and localized on actin filaments (Scale bar: $10 \mu \mathrm{m}$ ). 

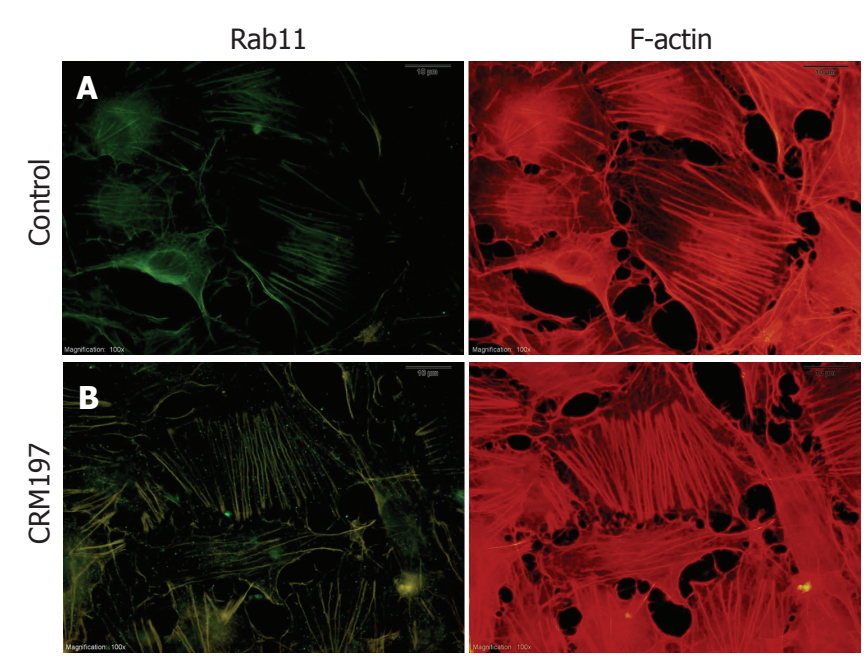

FIGURE 5. Transport of CRM197 along recycling pathway. For Rab11 whose receptor-mediated transporters were directed to recycling pathway, endothelial cells were labelled with anti-Rab11 (1:200) antibodies, and made visible with the aid of FITC-conjugated secondary antibodies (green). Actin filaments were labelled with phalloidin-TRITC (red). Prepared slides were covered with protective ProLong Gold Antifade. As shown in Figure 5, when control HUVEC (A) cells were compared with CRM197-treated endothelial cells (B) early endosomes carrying Rab11 peptide emitted stronger fluorescence signal in endothelial cells incubated with CRM197, and endosomes positioned together with actin filaments are detected (Scale bar: $10 \mu \mathrm{m}$ ).

membrane was labeled. When compared with control endosomes, Rab11 signal was detected stronger in CRM197loaded endosomes that were observed localized on actin filaments. As a result of labeling Rab7 which is the signal peptide for lysosomal pathway, any difference between the control, and CRM197-loaded endosomes could not be detected.

\section{DISCUSSION}

Actin plays an important role in the endocytic pathway during endocytosis of some pathogens, and their transport to targeted organelle or cell compartment. It has been suggested that actin cytoskeleton may act as a precursor in the very rapid transport of diphtheria toxin into perinuclear area with the aid of endosomes, and also considered that actin-FA-eEF2 interactions regulate ADP-ribosyltransferase activity. In our previous studies, the impact of both eEF2, and actin on transport of FA into cytosol was detected [8]. As a result of actin-FA-eEF2 interactions, it has been determined that ADP-ribosylation of eEF2 limits translocation of FA. Moreover depolymerization, and then degradation of actin filaments were demonstrated [9, $10]$. Following our previous investigations performed concerning the interaction between diphtheria toxin, and actin, in this study endocytic process of, CRM197, used in the production of pediatric vaccine, was analyzed. Intracellular trafficking of CRM197 was investigated in HUVECs. To determine the incubation time with CRM197, and then to show CRM197-loaded endosomal fraction at first stage DTx was used. In ultrastructural analysis, clustering of enlarged endosomes was determined around the nuclei of DTx-treated endothelial cells [17]. It has been revealed that FA in endosomal fractions obtained from cells incubated with DTx for 15 minutes, did not pass into cytosol, or even in conditions where actin cytoskeleton degraded, FA was trapped in early endosomes. Immunofluorescence method demonstrated that cargo-loaded endosomes spread through all of endothelial cells during endocytic process of CRM197. By the means of Western Blot it is determined that in CRM197-loaded endosomes, during the stage where FA does not leave endosomes, Hsp90, and actin molecules which will support entry of FA into cytosol also accompany endosomes. Findings indicate that early endosomes fused with CRM197-loaded endocytic vesicles tend to lead to recycling pathway.

During endocytic process, delivery of FA from endosomes depends on the presence of eEF2 as for both DTx, and CRM197 was shown [8]. Additionally, as demonstrated in many studies, cytosolic factors as Hsp90, and thioredoxin reductase also support translocation of FA [18]. Besides under the impact of VEGF in vascular endothelial cells, the role of Hsp90 played in the transmission of the cell migration signals into focal adhesion kinases has been also determined [19]. In our studies on CRM197-actin interaction it has been detected that 18 hours of contact with CRM197 caused disruption of $65 \%$ of F-actin in post-microsomal pellets, and intercellular contacts decreased in HUVECs when incubation time with CRM197 extended to 24 hours $[1,12]$. Simulation modelling of molecular dynamics has demonstrated that cysteins, localized on T-domain of CRM197 are amino acids most probably interact with actin [12]. Interaction between $\mathrm{T}$-domain and actin tends to support FA delivery over endosomal membrane into cytosol. Actin and Hsp90 identified on cell lysate fractions where CRM197-loaded endosomes are localized signify that FA has not been delivered from endosomes yet.

Rab11 was reported as one of the Rab proteins which direct intracellular trafficking of endosomes, also regu- 
late dynamics of actin cytoskeleton developed during cellular movements, and activity of RAC (small GTPase) [20]. More powerful signals produces by Rab11 peptide in CRM197-treated endothelial cells suggest that endosomes are directed again towards cell membrane by means of recycling pathway. Cargo-loaded early endosomes in CRM197-treated HeLa cells enlarged with successive fusions, but they did not transform into late endosomes which indicate lysosomal pathway [21]. In studies where CRM197 was used as drug delivery fusion protein, it was demonstrated that in common cell cultures, liposomes conjugated with CRM197 passed through from brain endothelial cell into astrocytes [22]. Drugs delivered by CRM197 carrier protein from endothelial cells which form blood-brain barrier are directed from apical towards basal lobes, thus delivery of drugs into brain tissue is achieved through transcytosis has been accepted. Delivery of lopermide into neurons was observed with in vivo studies by means of the strategy of overpassing blood-brain barrier using CRM197 nanoparticles [23].

CRM197-actin interaction, and leading of CRM197loaded endosomes towards cell membrane, before lysosomal pathway demonstrate compliance with the delivery strategy of drugs transported from brain endothelial cells by CRM197. In conclusion, we suggest that endocytic process of CRM197-loaded early endosomes in HUVECs progresses on the recycling pathway.

Acknowledgement: We would like to thank L. Ebru Akyon working at Department of Histology and Embryology, Istanbul Faculty of Medicine for her support in ultrastructural analysis.

Conflict of Interest: No conflict of interest was declared by the authors.

Financial Disclosure: This work was supported by the Scientific Research Project Coordination Unit of Istanbul University. Projects number: 21270, 39536 and 24735.

Authorship contributions: Concept - B.O.E., B.V; Design - B.O.E, E.H., M.B.; Supervision - M.B.; Materials - B.O.E, E.H.; Data collection \&/or processing - B.O.E, E.H., B.V.; Analysis and/or interpretation B.O.E., E.H., M.B.; Writing - B.O.E, E.H., B.V.; Critical review - M.B.

\section{REFERENCES}

1. Varol B, Özerman Edis B, Bektaş M. Toxin Structure, Delivery and Action. In: Burkovski A, editor. Corynebacterium diphtheriae and Related Toxigenic Species. Dordrecht: Springer Netherlands; 2014. p. 83-94.

2. Uchida T, Pappenheimer AM Jr, Greany R. Diphtheria toxin and related proteins. I. Isolation and properties of mutant proteins serologically related to diphtheria toxin. J Biol Chem 1973;248:3838-44.

3. Shinefield HR. Overview of the development and current use of CRM(197) conjugate vaccines for pediatric use. Vaccine 2010;28:4335-9.
4. Giannini G, Rappuoli R, Ratti G. The amino-acid sequence of two nontoxic mutants of diphtheria toxin: CRM45 and CRM197. Nucleic Acids Res 1984;12:4063-9. [CrossRef]

5. Van Ness BG, Howard JB, Bodley JW. ADP-ribosylation of elongation factor 2 by diphtheria toxin. NMR spectra and proposed structures of ribosyl-diphthamide and its hydrolysis products. J Biol Chem 1980;255:10710-6.

6. Naglich JG, Metherall JE, Russell DW, Eidels L. Expression cloning of a diphtheria toxin receptor: identity with a heparin-binding EGF-like growth factor precursor. Cell 1992;69:1051-61. [CrossRef]

7. Lemichez E, Bomsel M, Devilliers G, vanderSpek J, Murphy JR, Lukianov $\mathrm{EV}$, et al. Membrane translocation of diphtheria toxin fragment A exploits early to late endosome trafficking machinery. Mol Microbiol 1997;23:44557. [CrossRef]

8. Bektaş M, Hacıosmanoğlu E, Ozerman B, Varol B, Nurten R, Bermek E. On diphtheria toxin fragment $\mathrm{A}$ release into the cytosol--cytochalasin $\mathrm{D}$ effect and involvement of actin filaments and eukaryotic elongation factor 2. Int J Biochem Cell Biol 2011;43:1365-72. [CrossRef]

9. Bektaş M, Varol B, Nurten R, Bermek E. Interaction of diphtheria toxin (fragment A) with actin. Cell Biochem Funct 2009;27:430-9. [CrossRef]

10. Varol B, Bektaş M, Nurten R, Bermek E. The cytotoxic effect of diphtheria toxin on the actin cytoskeleton. Cell Mol Biol Lett 2012;17:49-61.

11. Unlü A, Bektaş M, Sener S, Nurten R. The interaction between actin and FA fragment of diphtheria toxin. Mol Biol Rep 2013;40:3135-45.

12. Özerman Edis B, Varol B, Hacıosmanoğlu E, Ünlü A, Bektaş M. Crossreacting material 197 (CRM197) affects actin cytoskeleton of endothelial cells. Gen Physiol Biophys 2017;36:383-9. [CrossRef]

13. Scita G, Di Fiore PP. The endocytic matrix. Nature 2010;463:464-73.

14. Oh KJ, Senzel L, Collier RJ, Finkelstein A. Translocation of the catalytic domain of diphtheria toxin across planar phospholipid bilayers by its own T domain. Proc Natl Acad Sci U S A 1999;96:8467-70. [CrossRef]

15. Tjelle TE, Brech A, Juvet LK, Griffiths G, Berg T. Isolation and characterization of early endosomes, late endosomes and terminal lysosomes: their role in protein degradation. J Cell Sci 1996;109:2905-14.

16. Laemmli UK. Cleavage of structural proteins during the assembly of the head of bacteriophage T4. Nature 1970;227:680-5. [CrossRef]

17. Morris RE, Gerstein AS, Bonventre PF, Saelinger CB. Receptor-mediated entry of diphtheria toxin into monkey kidney (Vero) cells: electron microscopic evaluation. Infect Immun 1985;50:721-7.

18. Ratts R, Zeng H, Berg EA, Blue C, McComb ME, Costello CE, et al. The cytosolic entry of diphtheria toxin catalytic domain requires a host cell cytosolic translocation factor complex. J Cell Biol 2003;160:1139-50.

19. Rousseau S, Houle F, Kotanides H, Witte L, Waltenberger J, Landry J, et al. Vascular endothelial growth factor (VEGF)-driven actin-based motility is mediated by VEGFR2 and requires concerted activation of stress-activated protein kinase 2 (SAPK2/p38) and geldanamycin-sensitive phosphorylation of focal adhesion kinase. J Biol Chem 2000;275:10661-72.

20. Ramel D, Wang X, Laflamme C, Montell DJ, Emery G. Rab11 regulates cell-cell communication during collective cell movements. Nat Cell Biol 2013;15:317-24. [CrossRef]

21. Antignani A, Youle RJ. Endosome fusion induced by diphtheria toxin translocation domain. Proc Natl Acad Sci U S A 2008;105:8020-5.

22. Gaillard PJ, de Boer AG. A novel opportunity for targeted drug delivery to the brain. J Control Release 2006;116:e60-2. [CrossRef]

23. Tosi G, Vilella A, Veratti P, Belletti D, Pederzoli F, Ruozi B, et al. Exploiting Bacterial Pathways for BBB Crossing with PLGA Nanoparticles Modified with a Mutated Form of Diphtheria Toxin (CRM197): In Vivo Experiments. Mol Pharm 2015;12:3672-84. [CrossRef] 